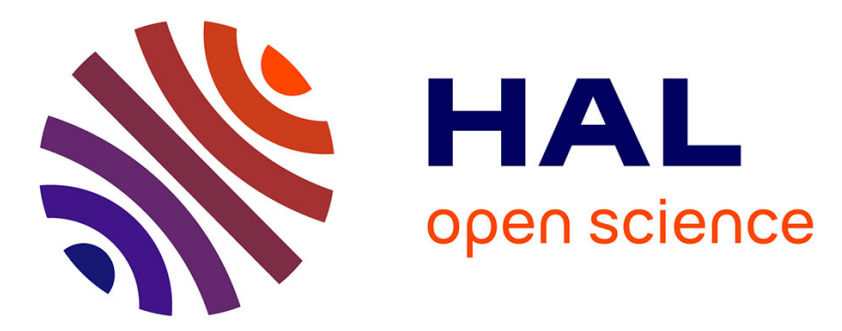

\title{
La "virtus universalis": un concept d'origine hermétique? Les sources d'une notion de philosophie naturelle médiévale
}

\author{
Isabelle Draelants
}

\section{- To cite this version:}

Isabelle Draelants. La "virtus universalis": un concept d'origine hermétique? Les sources d'une notion de philosophie naturelle médiévale. P. LUCENTINI - I. PARRI - V. PERRONE COMPAGNI, éd., Hermetism from late antiquity to humanism. La tradizione ermetica dal mondo tardo antico all'Umanesimo, Atti del Convegno Internazionale di Studi (Napoli, 20-24 nov. 2001)„ 40, pp.157-188, 2003, Instrumenta patristica et mediaevalia, 2-503-51616-5. halshs-03096170

\section{HAL Id: halshs-03096170 \\ https://shs.hal.science/halshs-03096170}

Submitted on 4 Jan 2021

HAL is a multi-disciplinary open access archive for the deposit and dissemination of scientific research documents, whether they are published or not. The documents may come from teaching and research institutions in France or abroad, or from public or private research centers.
L'archive ouverte pluridisciplinaire HAL, est destinée au dépôt et à la diffusion de documents scientifiques de niveau recherche, publiés ou non, émanant des établissements d'enseignement et de recherche français ou étrangers, des laboratoires publics ou privés. 
La "virtus universalis": un concept d'origine hermétique? Les sources d'une notion de philosophie naturelle médiévale

\section{Isabelle Draelants}

Si l'on a beaucoup étudié la prospérité médiévale du dialogue hermétique de l'Asclepius, si l'on connaît bien le Liber viginti quattuor philosophorum et depuis peu le Liber de sex rerum principizs, c'est récemment néanmoins que la recherche relative aux autres ouvrages mis sous le nom d'Hermès au Moyen Âge a pris un tournant décisif - principalement d'ailleurs sous l'égide de l'institution qui accueille ce colloque. Le concept étudié dans cette contribution devrait permettre de mettre sur la piste de l'un de ces ouvrages perdus ou à redécouvrir, tout en illustrant la circulation de l'hermétisme dans l'Occident latin, non pas dans ses manifestations théologiques, mais dans une de ses applications en philosophie naturelle.

Au XIII ${ }^{\mathrm{e}}$ siècle, plusieurs œuvres de philosophes intéressés à la nature et à ses lois recourent au concept de virtus universalis, à propos d'une force qui agit et se diffuse dans la nature et permet d'expliquer l'action particulière de certaines substances. En parallèle, une force proche, assimilable peut-être à la "vertu formative", est désignée par vis mineralis. Les deux expressions se valent-elles ou ont-elles été confondues à tort? Renvoient-elles à une notion nouvelle et 'inventée' au XIII ${ }^{\mathrm{e}}$ siècle, ou bien au contraire désignet-on ainsi une doctrine déjà présente, soit dans la littérature latine antérieure, soit dans la tradition des textes hérités de l'Antiquité via les Arabes? Autant de questions qui doivent ètre abordées, quand on constate que ces notions se répandirent dans la littérature philosophique et didactique au cours des deux siècles suivants. Un champ compliqué de significations, du fait de racines philosophiques multiples et d'applications variées, est né de ces concepts. A partir d'un cas précis, il s'agit ici d'expliquer le concept de “vertu univer-

* Merci à Anka Krivat et à David Juste d'avoir bien voulu relire une version précoce de cet article avec une attention amicale. 
selle" et de présenter certaines sources textuelles de son intégration dans la philosophie naturelle médiévale; l'une d'entre elles porte le nom d'Hermès.

Parmi les premières attestations, la virtus universalis apparaît déjà assimilée, avant le milieu du siècle, dans une encyclopédie de philosophie naturelle et morale, le De floribus rerum naturalium d'Arnold de Saxe ${ }^{1}$. A peu près concommitamment, on la retrouve sous diverses formes dans plusieurs des premières œuvres philosophiques d'Albert le Grand: la Summa de creaturis, le De natura loci, le commentaire sur la Physique et le De mineralibus qui s'inspire du "Traité des pierres" d'Arnold Saxo. On rencontre ensuite des concepts apparentés dans le Breve Breviarium attribué à Roger Bacon, la Summa philosophiae du Pseudo-Grosseteste, et, bien sûr, le De occultis operationibus naturae de Thomas d'Aquin. Il est aisé de reconnaître, dans la plupart de ces attestations, une filiation Arnold Saxo - Albert le Grand, puis une diffusion qui doit beaucoup au rayonnement $\mathrm{du}$ traité sur les pierres du doctor universalis. On le constate d'emblée, les ouvrages cités s'intéressent avant tout à la nature et s'inspirent d'un corpus qui s'est constitué à partir des ceuvres d'Aristote traduites au XII ${ }^{\mathrm{e}}$ siècle, mais aussi des commentaires et traités arabes qui les accompagnaient.

1. Littérature médicale ou 'd'expérience': Avicenne, Aristote et les propriétés naturelles.

Une attestation explicite de la virtus universalis figure dans l'encyclopédie naturelle et morale écrite par Arnold Saxo. Contemporain et peut-ètre collaborateur d'Albert le Grand, il porta un vif intérêt à la médecine, au point de rédiger une Practica à la fin de sa vie, probablement autour de $1270^{2}$. Dans cet ouvrage, il tient

1 Cet auteur a fait l'objet de ma thèse de doctorat, cf. Draelants 2000a. Je prépare une édition critique du De floribus rerum naturalium (Brepols). En attendant: Arnoldus Saxo 1905. Pour un aperçu des œuvres et des sources d'Arnoldus Saxo, Draelants 2001; Draelants 1992; Draelants 1993. Dans les manuscrits, on trouve indifféremment "Arnoldus Saxo" ou "Arnoldus Luca".

2 Cette datation repose en partie sur le fait que l'unique manuscrit conservé de la Practica de curis morborum contient des extraits du commentaire à l'Antidotarium Nicolai de Jean de Saint-Amand, rédigé au plus tòt autour de cette date. En l'absence d'autres manuscrits de la Practica, l'hypothèse d'une révision postérieure à la rédaction par Arnold Saxo n'est pas à rejeter a priori, mais elle parait peu vraisemblable. compte de l'évolution des connaissances médicales de son temps. Au début de son activité intellectuelle, entre 1230 et 1245 , il rassembla un grand nombre de sources philosophiques et scientifiques issues $\mathbf{d u}$ corpus arabo-latin pour rédiger une encyclopédie-florilège en cinq parties, le De floribus rerum naturalium ${ }^{3}$. Elle comporte un tiers de citations d'Aristote et beaucoup d'extraits pseudépigraphes mis sous son nom. La quatrième partie de l'œuvre, assez courte, a justement pour titre De virtute universali; elle décrit les êtres qui peuplent le monde physique à partir de ce principe d'explication: l'homme, les animaux sauvages et domestiques, les oiseaux, les poissons, les reptiles, les plantes, les pierres, auxquels sont ajoutés - sans doute dans une deuxième étape de compilation - des principes d'optique. Le texte est constitué d'une mosaïque d'extraits qui reconstituent environ cent soixante nouvelles unités de citation. Galien et Avicenne y côtoient des sources plus obscures relatives aux propriétés médicomagiques des substances animales, végétales et minérales, d'un contenu qu'on peut comparer à celui des Kyranides. S'ajoutent à ces textes pseudépigraphiques attribués à Pythagoras, Esculapius, Zeno ou Balīnūs ${ }^{4}$, des citations des Parva naturalia d'Aristote et des extraits de deux versions concurrentes du Livre des pierres qui est attribué à ce dernier. Voici comment Arnold Saxo définit la vertu universelle dans le prologue à cette partie de son œuvre intitulée De virtute universali:

Nunc vero, que a virtute universali seu a tota substantia vel a forma specifica sunt operationes a pluribus philosophis collectas ... ordinavi. Unde librum De virtute universali in $\mathrm{X}$ capitula iam distinxi. A sua quidem operans substantia seu a virtute universali, est illud, quod forma sue speciei operatur, quam acquisivit post complexionem, cum eius simplicia se commiscuerunt; et ex eis generata fuit res una, sicut virtus attractiva, que est in magnete, et sicut natura cuiusque specierum vegetabilium et animalium illa, quam habent post complexionem. Neque est complexio simplex vel composita, sed est, verbi gratia, color aut odor aut anima aut alia forma non perceptibilis unquam sensu.

Il s'agit donc de mettre en évidence les opérations propres à chaque créature, en fonction de sa "substance tout entière" ou de sa "forme spécifique", ou de "la vertu universelle". Ces termes semblent jouer ici comme des synonymes. Ces notions sont utilisées

3 Cf. Draelants 2000a; Draelants 2001

${ }^{4}$ Cf. Draelants 2000a, pp. 325-360. 
par Arnold Saxo pour montrer comment agissent les propriétés de la chair de tel animal, de la plante ou de la pierre et dans quel cas ces propriétés sont applicables. Il avance ainsi qu'une propriété particulière peut naitre d'une sorte de "composition" individuelle propre à une espèce. Le mème objectif est mentionné déjà dans le prologue à la deuxième partie, Sur les natures des animaux, où Arnold dit: "Librum de naturis animalium iam composui, cum diversitate plurima, que a virtute universali vel secundum naturam sunt singulis animalibus attributa".

Les concepts utilisés ici - opération, substance, forme spécifique, vertu - relèvent de la physique des éléments, mais ils font auss partie des instruments de la théorie médicale tout autant que de l'arsenal philosophique répandu alors. La réunion des différents termes n'est pas une création du compilateur: la source de cette étrange affirmation peut être en effet trouvée, presque mot pour mot, dans le Canon d'Avicenne. On y lit que la "forme spécifique" liée à toute la substance apparaît après le mélange des éléments simples et qu'elle s'ajoute aux formes qu'ont les corps simples. Cette perfection acquise par la matière provient d'une aptitude - donc d'une vertu - due à la complexion, qui permet d'expliquer l'action d'attraction étrange de l'aimant.

Et sua quod operans substantia est illud quod forma sue speciei operatur quam acquisivit post complexionem quod cum eius simplicia se comiscuerunt et ex eis generata fuit res una preparavit se ad recipiendum speciem et formam additam super illud quod habent simplicia illius.

Hec ergo forma non est qualitates prime quas habet materia: neque est complexio qua generatur ex eis: sed est perfectio quam acquisivit materia secundum aptitudinem que fuit ei acquisita ex complexione sicut in magnete virtus attractiva: et sicut natura cuicumque specierum vegetabilium et animalium ... quia non est caliditas, neque frigiditas, neque siccitas, neque humiditas, neque simplices neque commixte, sed est verbi gratia color aut odor, aut anima aut alia forma de non preceptis sensus ${ }^{5}$.

Chez Arnold Saxo, l'application de la virtus universalis à la "nature des choses" s'appuye d'emblée sur un texte médical arabe de pharmacopée, mais l'initiative d'assimiler la "vertu universelle", absente du texte d'Avicenne, à la "substance tout entière" est-elle

\footnotetext{
${ }^{5}$ Avicenna 1507, f. 33a [I, 2, 2, 1, 15].
}

due à l'encyclopédiste également ou à une autre de ses sources restant à découvrir?

Avant de rechercher celle-ci, examinons le contexte de l'emploi du concept de "forme spécifique" et ses liens avec la "substance tout entière", puisque dans le texte, le concept de tota substantia parait se fondre dans celui de tota species ou de forma specifica. Arnold souligne que les actions réalisées par la forme spécifique ont pour particularité de se passer de l'intervention d'une qualité intermédiaire. C'est bien le sens de la suite du texte d'Avicenne, qui souligne que cette opération particulière a lieu par "toute la substance", c'est-à-dire par la "forme spécifique" et non par une des quatre qualités. La postérité médicale de cette notion fut réelle, puisque Lynn Thorndike ${ }^{6}$, Nancy Siraisi et Danielle Jacquart ont montré que le concept de forme spécifique est invoqué à la fin du XIII ${ }^{\mathrm{e}}$ siècle et au siècle suivant chez des médecins comme Turisanus, Taddeo Alderotti ou Pietro d'Abano pour expliquer un troisième type d'attraction, 'occulte', par opposition à l'action manifeste de certaines substances ${ }^{7}$.

Chez le médecin montpelliérain Arnaud de Villeneuve, on trouve au tournant des $\mathrm{XIII}^{\mathrm{e}}$ et $\mathrm{XIV}^{\mathrm{e}}$ siècles une mise en contexte explicite de la "forme spécifique" et de la "vertu occulte" qui en est issue, où le passage d'Avicenne - emprunt inavoué - soutient le raisonnement médical. Il s'agit d'un long développement sur la manière de soigner en fonction de la complexion du patient, dans le Speculum medicinae ou Speculum introductionum medicinalium. En mentionnant l'exemple particulier des minéraux, il définit la propriété occulte comme n'étant pas perceptible par les sens comme le sont la chaleur, le froid, la couleur, l'odeur et le goùt; c'est la raison pour laquelle on ne peut la quantifier. Son existence ne peut ètre reconnue par l'expérience de la raison, mais seulement par une décou-

6 Thorndike 1923-1958, I, pp. 644-646 (al-Kindĩ et les rayons célestes); II, pp. 209-210 (Maimonides); pp. 535, 563-569 (Albert le Grand); pp. 854-859 (Arnauld de Villeneuve). Aux pp. 906-910, il traduit la définition suivante de la forme spécifique, d'après Pierre d'Abano: "It is nothing else than the value or valence (merilum) which any object composed of the four elements acquires from the proportions of those elements existing in this compound and from the influence of the fixed stars which regards the species of inferior compounds".

${ }^{7}$ Siraisi 1981, pp. 295-296 surtout; Jacquart 1998, pp. 371-377. Le passage d'Avicenne est commenté aussi par Weill-Parot 2002, pp. 450-452. 
verte par hasard. C'est dû au fait qu'une telle vertu occulte dépend de deux choses: le mélange des éléments dans l'objet qui la possède et sa "forme spécifique" 8 .

Activarum vero virtutum quedam sunt communes omnibus complexionatis et hee sunt quatuor qualitates prime quas omnis medicus ... vocat activas. Alie vero sunt proprie et ideo proprietates dicuntur et a quibusdam virtutes specivoce ${ }^{9}$ quia formam specivocam consequuntur. Similiter etiam quia tales virtutes communes sunt omnibus individuis speciei propterea operatio ipsarum dicitur a tota specie fieri. Et quidam dicunt a tota substancia propterea quia rei convenit racione eius quod est perfectio substancie tocius compositi. Ex hiis ergo patet quod proprietas est virtus que debetur complexionato ratione eorum que propria sunt ipsius. Hec autem sunt duo scilicet forma specivoca et gradus mixtionis disponens ad illam formam ... Que licet proprietates innominate sint a suis tamen operationibus quibus cognoscuntur denominationem recipiunt ut in magnete que dicitur tractiva ferri ... Multi medicorum proprietatem vocaverunt virtutem occultam ... Ex quo etiam patet quod non ideo dicitur occulta quia minus cognoscatur suo effectu quam virtus complexionalis ... Sed pro tanto dicitur occulta quoniam ea quibus res cognoscitur omnino sunt aput humanam rationem ignota. Omnis enim res determinate cognoscitur vel a causis immediatis vel effectu immediato quorum utrumque in proprietatibus ignorat humana ratio. Cause enim immediate proprietatum ut dictum est due sunt scilicet forma mixtionis et forma specivoca forma enim mixtionis ex quantitate miscibilium ... ad quorum noticiam pervenire non potest humana ratio cum hec in infinitum varientur. Nec per consequens ad formam specivocam que ex potencia materie consurgit. Effectum etiam proprietatis nequit ratione cognosci cum priora in eis sint occulta nec etiam colligi potest rationali experimento sed tantummodo casuali Rationale ... Dicitur enim occulta quoniam ipsa in se non est sensum perceptibilis sicut actualis caliditas vel frigiditas aut color aut odor <aut sapor> et universaliter omnes sensibiles qualitates.

Définissant la notion médicale de "vertu", Avicenne avait en effet lié la forme spécifique à la propriété propre à l'espèce, expliquant ainsi la vertu d'attraction occulte. Le raisonnement s'est inscrit naturellement dans la tradition de la médecine salernitaine du XII siècle, puisqu'il complétait la notion fondamentale de "complexion",

8 Arnaldus de Villanova 1495, f. Diiir-iiiv [7]. Correspond au c. 18 dans Arnaldus de Villanova 1509, f. 6vb.

9 'Specivocus-a-um': lege 'specificus-a-um'. qui désigne un mélange de qualités premières propres à tout corps. La notion de complexion, elle-même véhiculée par la médecine galénique adaptée par les Arabes, permettait de relier la physiologie à la lointaine physique élémentaire aristotélicienne, où le changement physique était régi par l'interaction des quatres qualités fondamentales (chaud, froid, sec et humide). Deux éléments pris ensemble peuvent devenir un troisième, en perdant chacun une qualité et à condition que celles qui restent, de part et d'autre, ne soient pas incompatibles (contraires). La "forme" seule subit donc le changement alors que la "matière" première reste fondamentale ${ }^{10}$. Tout en spécialisant cette théorie, la médecine mettait en évidence le rapport entre les principes cosmologiques et les composants spécifiques des corps que sont les quatre humeurs.

Assimilée à ce système axé sur la réalité sensible et fondé sur les couples matière/forme et substance/propriété, la théorie d'Avicenne permit d'appréhender rationnellement les causes 'occultes' ou imperceptibles par les sens. Dans la pratique, la médecine avait pour objet de manipuler la vertu propre à une substance, vertu dont il fallait découvrir la cause ou du moins décrire l'origine pour comprendre toute formation (generatio) ou transformation (operatio) physique. C'est ainsi que, dans la médecine pénétrée de magie des Arabes puis des Latins, certains remèdes ne pouvant être déduits de la composition élémentaire des substances étaient connus comme éprouvés par l'expérience (c'est-à-dire par leur effet) ${ }^{\prime \prime}$. Les propriétés particulières y étaient présentées comme attachées à quelque entité qui représentait l'individualité du corps qui les produisait, comme la "forme substantielle" de ce corps ou sa "complexion", ou son "tempérament"; on disait qu'elles opéraient "avec toute leur substance" ${ }^{12}$. Cette "forme spécifique" (ou substantielle) s'identifiait u mélange (commixtio) d'éléments purs (simplicia commixerunt), qu'on situait à un niveau supérieur au niveau élémentaire: celui

10 Sur les notions propres à la science de la matière en rapport avec la contagion et l'infection, voir p. ex. Dales 1972

11 Dans ses consilia Taddeo Alderotti fait une différence claire, vers 12601270 , entre les médicaments trouvés "par la raison" et ceux qui le sont "par l'expérience". Cf. Siraisi 1981, p. 294 n. 92, où elle cite ceci: "Viso de cura per rationem, videndum est de cura per empirica experimenta, per formam specificam operantia".

12 Hutchison 1982, p. 240. 
des corps mixtes comme les plantes, les pierres ou les animaux. Elle leur permettait une action (vertu) spécifique, par exemple attractive, en médecine. Comme ces propriétés particulières, sympathiques, des substances semblaient non naturelles, on avançait le plus souvent pour les illustrer l'exemple de l'attraction de l'aimant, qui devint courant chez les médecins latins pour aborder des phénomènes vérifiables seulement par l'expérience.

Sans toucher encore là l'origine précise du terme, on constate déjà que le contexte de l'utilisation de la "vertu universelle" plonge ses racines dans les textes de tradition médico-magique arabe inclus dans la philosophia naturalis et qu'elle a ouvert la voie à ce qu'on peut appeler la 'littérature d'expérience' dans le monde occidental latin. A partir de la seconde moitié du XIII ${ }^{\mathrm{e}}$ siècle, cette théorie de la nature relative à la propriété particulière d'une substance a favorisé en effet la production de recueils appelés Secreta ou Experimenta, consacrés aux propriétés occultes des choses reconnaissables seulement par l'expérience et non par la connaissance intellectuelle "sensible" ${ }^{13}$. Autrement dit, ces textes s'intéressent aux effets spécifiques des naturalia - animaux, plantes ou pierres - qui apparaissent séparés de leur cause et ne sont pas distribués universellement dans la nature ni réductibles aux caractéristiques universelles des quatre éléments. Un des premiers, sinon le premier des traités de ce genre, fut attribué dès l'origine à Albert le Grand: le Liber aggregationis ou De proprietatibus herbarum, lapidum et animalium, encore appelé Experimenta Alberti ${ }^{14}$. Comme on va le voir, plusieurs des ouvres authentiques de philosophie naturelle contiennent chez Albert les éléments compatibles avec cette conception des choses. Certaines d'entre elles comptent parmi leurs sources l'information apportée par Arnold Saxo et y ajoutent des éléments explicites, susceptibles de mener à d'autres identifications de sources.

13 Sur la différence entre la connaissance sensible et l'expérience, voir Hutchison 1982, pp. 239-240.

14 Albertus Magnus 1973. Sur les 'livres des secrets' en général, cf. Eamon 1985 et Eamon 1994. Le Liber aggregationis fait l'objet d'une bourse de recherche (Frances Yates fellowship), que je mène au Warburg Institute de Iondres (avriljuillet 2002) et que fera l'objet d'une édition critique.
2. Minéralogie et botanique: Hermès, Aristote et la causalité céleste.

a) Chez Albert le Grand et Arnold Saxo: le De virtute universal d'Hermès.

La quête de faits d'expérience va, chez Albert le Grand, de pair avec la recherche des fondements philosophiques - c'est-à-dire des causes des phénomènes. Il n'est donc pas étonnant de le voir expliquer par la forme substantielle ou spécifique les propriétés particulières de la matière ${ }^{15}$, mais il est plus novateur de souligner, dans certaines de ses œuvres, l'utilisation de la "vertu universelle".

Lynn Thorndike avait déjà relevé dans le De vegetabilibus d'Albert l'affirmation selon laquelle les propriétés des plantes étaient produites par la combinaison des vertus suivantes: celle de l'élément qui prédomine dans la complexion de la plante, la vertu des autres éléments qui sont mélangés avec le premier, l'influence des étoiles et la vertu de l'âme végétale ${ }^{16}$. La forme spécifique, disait la suite du texte, était responsable en grande partie des vertus occultes de la plante, qui dépendaient du mouvement céleste au moment de la formation de la plante. Déjà dès ses premières œuvres (qui coïncident avec le témoignage d'Arnold Saxo examiné plus haut), Albert avait avancé ce raisonnement de l'action spécifique en ce qui concerne les médicaments.

Et ad hoc intelligendum, notandum quod transmutatio corporum est quatuor modis: quorum unus est miscibilium ad actum mixti tantum, in quo miscibilia remanent secundum veritatem consequentem suas proprias species, licet non remaneant secundum formam suarum specierum, sicut miscentur medicinae compositae, ut tiriaca, et eluctuaria, et huiusmodi: et in talibus quaedam sunt virtutes, quae consequuntur singula recepta, et natura quae sagax est, quando accipiuntur dividit ea ${ }^{17}$.

15 On renverra utilement aux acquis de Jeck 1994. Il a montré, chez Albert le Grand, l'émergence d'une nouvelle conception de la matière et de l'espèce qui remonte à ses premiers écrits de philosophie naturelle, comme la Summa de creaturis. D'après lui (p. 210), Albert a assimilé les concepts de materia et de forma substantialis d'après Aristote, celui de species d'après Augustin. Ensuite, il aurait fait de la forma substantialis une sorte de traduction des idées platoniciennes sous forme de matière (pp. 213-214).

16 Thorndike 1923-1958, II, pp. 565-566, citant Albert le Grand, De vegetabilibus, V, II, 1. La suite, à propos de la forme spécifique, se trouve dans le De vegetabilibus, VI, II, 22.

${ }^{17}$ Albertus Magnus 1867, p. 155b [7, 8] (cité par Jeck 1994, p. 223 n. 50). 
Par ailleurs, les effets occultes des plantes et des pierres renforcés par les ligatures sont admis par Albert, tant dans le De mineralibus que dans le De vegetabilibus. Ils le sont par le biais de "l'expérience" - ce qui signifie, dans la mentalité médiévale, qu'un jour ils ont été observés par un philosophe dont l'autorité est digne de confiance. En l'occurrence, les auctoritates alléguées par Albert le Grand à propos du pouvoir des pierres portées en talismans sont, entre autres, Thābit ibn Qurra, Ptolémée, Constantin l'Africain (qui traduisit au Mont-Cassin le De physicis ligaturis de Qusțā ibn Lũqā, qu'Albert mentionne également ${ }^{18}$ ), et Hermès le philosophe.

- Nunc autem determinemus causam quare gemmae primitus a sapientibus sculpi praeceptae sunt, et quod sit iuvamentum in ipsis sigillis eorum. Huius autem causam cognoscere ex scientia oportet magorum quam compleverunt Magor Graecus, et Germa Babylonicus, et Hermes Aegyptius in primis, postea autem mirabiliter effulsit in ea Ptolemaeus sapiens et Geber Hispalensis, Tebith autem plene tradidit artem. Est autem principium in ipsa scientia omnia quaecumque fiunt a natura vel arte, moveri a virtutibus caelestibus primo: et hic de natura non est dubium. In arte etiam constat, eo quod aliquid modo et non ante incitat cor hominis ad faciendum: et hoc esse non potest nisi virtus caelestis, ut dicunt sapientes praenominati ${ }^{19}$.

- Et sunt multae tales de quibus scribitur in libris incantationum Hermetis Philosophi, et Costebenluce Philosophi, et in libris de physicis ligaturis inscriptis ${ }^{20}$.

Par rapport aux témoignages précédents, la causalité céleste apparait ici comme un élément nouveau dans l'explication des propriétés. Elle est évidemment à l'œuvre lors de la confection d'images astrologiques censées conférer à l'objet sur lequel elles figurent (pierre ou autre support) une vertu particulière issue de la vertu céleste ${ }^{21}$. En rassemblant diverses informations trouvées dans la documentation philosophique disponible, Albert le Grand nous en

${ }^{18}$ Le nom "Costabulenca" renvoie à Qusțã ibn Lũqã, l'auteur arabe (IX ${ }^{\mathrm{e}} \mathrm{s}$.) du traité sur les ligatures traduit par Constantin au XI ${ }^{\mathrm{e}}$ s. Cf. Wilcox 1995.

${ }_{19}$ Albertus Magnus 1890, p. 51 [II, 3]. Les noms de Thābit, Germa Babiloniensis et Ptolémée, dans leurs rapports avec les talismans minéraux, ont déjà été élucidés dans d'autres publications (notamment Sannino 2000; Lucentini 2000; Pingree 1987; Draelants $2000 \mathrm{a}$, pp. 488-495), c'est pourquoi je n'y reviens.

20 Albertus Magnus 1867 , p. 158b [V, 2, 6].
21 Cf. Weill-Parot 1999, pp. 217, 222 . apprend davantage sur l'origine et le sens de la virtus celestis dans la nature. Il l'insère dans la théorie générale des causes, et plus particulièrement la causalité céleste. Dans le chapitre qui traite de la cause générative des pierres, il dit avoir trouvé cette vertu exprimée et illustrée par l'exemple du soleil dans un ouvrage attribué à Hermès, et introduit les termes de virtus universalis déjà trouvés chez Arnold Saxo. Il avance que l'universalité de cette force, même si elle n'est pas toujours visible, est réelle en toute substance au même titre que le rayonnement du soleil. Faut-il pour autant rapporter la vertu universelle à la vertu céleste?

Hermes autem in libro quem de minerali (lege universali) virtute scribit, dicere videtur causam generativam lapidum esse virtutem quamdam, quam unam dicit esse in omnibus, sed propter diversitatem rerum generatarum diversa sortiri vocabula. Exemplum ponens de lumine solis, quod solum omnium est generativum, et cum participatur, non per unam solam passivorum potestatem, diversa operatur in eis ${ }^{22}$.

L'ouvrage d'Hermès est appelé, dit-il, De virtute universali ${ }^{23}$, c'est-à-dire le titre mème choisi par Arnold pour coiffer la quatrième partie du De floribus rerum naturalium ${ }^{24}$. Deux autres fois, en dehors du De mineralibus, Albert mentionne cet ouvrage (sous la forme Hermes in libro De virtutibus universalibus) dans une discussion sur la nature universelle ou particulière, où il avance l'exemple de la diffusion de la lumière du soleil comme une force venue du ciel: dans la Physica et dans le De natura loci ${ }^{25}$. Hermès, les Pythagoriciens et Platon sont allégués, en argumentant cependant qu'il ne s'agissait pas d'une force (vis), mais d'une substance, sinon elle ne pourrait se diviser, en provenance de la première cause, pour atteindre les choses inférieures à travers le mouvement du ciel. Comme l'a souligné $\mathrm{P}$. Lucentini, la nature universelle précèderait ainsi les natures particulières et se diviserait, ce qui combat la métaphysique

${ }^{22}$ Albertus Magnus 1890, p. 5 [I, 1, 4].

23 De par une mauvaise leçon de l'éd. Borgnet, la vertu universelle a disparu du texte d'Albert, mais plusieurs manuscrits attestent bien ici universali, comme l'édition d'Oppenheim de 1517 , en général assez fiable.

${ }^{24}$ A noter qu'aucune citation de cet 'ouvrage' d'Hermès n'apparaît comme telle chez Arnold.

${ }_{25}$ Albertus Magnus 1987, p. 83, 63/65 [11, 1, 5]; Albertus Magnus 1980, pp. 8, $78-9,12[\mathrm{I}, 5]$. Cf. texte cité ci-dessous, p. 168 . 
aristotélicienne ${ }^{26}$. Cette substance trouve le principe de son mouvement et de son repos dans toutes choses, mais elle peut ètre entendue aussi comme ce qui est déterminé dans le genre ou l'espèce, comme la nature animale ou humaine est dite universelle.

Et non est verum, quod dixerunt praenominati viri; dixerunt enim illi, quod natura est "vis" absoluta "diversificata in specie et diffusa in" particularibus illius speciei per "divisionem" sui in ipsis et quod "natura" absoluta est forma et vis quaedam egrediens a causa prima per motum orbis, quae postquam egressa est, "diffunditur in" omnibus naturalibus et fit in eis principium motus et status. Et posuerunt simile huius in lumine, quod egreditur de "sole", quod quidem "unum" est iuxta solem ${ }^{27}$.

Cette doctrine est proche de ce qu'on trouve exprimé dans le De sex rerum principiis hermétique: "Natura quoque vigor quaedam est, universalis et specialis, ex causa et racione nascens, primum celo innascens" ${ }^{28}$

Plus loin dans le De mineralibus, à propos de la cause de la vertu des pierres, Albert réaffirme l'importance de l'ouvrage de celui qu'il considère comme le plus ancien philosophe paien en matière de philosophie naturelle, Hermès.

Hermes autem et quidam sequaces eius Indorum plurimi multa et universali virtute differentes, dicebant omnium inferiorum virtutes esse in stellis et imaginibus coelorum ${ }^{29}$.

Si aucune œuvre latine intitulée De virtute universali n'a été signalée, il est incontestable cependant que les deux naturalistes, Arnold et Albert, se réfèrent à des sources identiques, qui mettent en évidence les sympathies occultes reliant les objets de la nature et les plaçant dans une dimension universelle. De nombreuses ouvres arabes attribuées à Hermès concernent ces correspondances. Toute la collection des Kyranides ${ }^{30}$ et des ouvrages apparentés, tels les

26 Sur ce sujet et ce passage, Lucentini 2000, p. 430; sur les conceptions hermétiques d'Albert, pp. 431-432.

27 Albertus Magnus 1987, p. 83 [I, 1, 5].

28 De sex rerum principiis 1955 , pp. 248-249.

29 Albertus Magnus 1890, p. 26 [II, 1, 2].

30 Voir Kyranides 1942, pp. 11-206; Kaimakis édite le texte grec: Kyraniden 1976. Thorndike 1923-1958, II, pp. 229-235, fait une courte étude de l'ouvrage; Festugière 1950-1954, I, pp. 201-216; Ganszyniec 1920-1921, pp. 353-369, 56-65, traités hermétiques sur les talismans, appartient à ce groupe dont des fragments, passés dans les compilations latines, prolongent audelà des siècles la tradition des Physika égyptiens (II ${ }^{\mathrm{e}}$ siècle a. C.) et mettent en évidence les liens occultes de similitude entre les pierres, les plantes et les animaux ${ }^{31}$.

Julius Ruska, étudiant la Table d'Emeraude, s'était demandé à propos d'Albert de quel texte il s'agissait, sans réussir à identifier un traité d'Hermès sous ce nom. Il a néanmoins fait le rapport de sens avec le Livre du secret de la créature d'Apollonius de Tyane ${ }^{32}$. Il $\mathrm{y}$ a incontestablement des liens de doctrine avec les traités liés à la Table d'Emeraude de ce magicien du premier siècle. D'ailleurs, Apollonius était cité sous le nom de Belenus dans le livre Sur la vertu universelle d'Arnold Saxo, c'est-à-dire sous la forme arabe (Balīnūs) de son nom. A ses còtés, on lisait vingt-cinq citations d'un ouvrage Sur les choses naturelles (De naturalibus) attribué à Zénon ${ }^{33}$. On ne s'étonnera donc pas de retrouver également cette dernière auctoritas au sein du De mineralibus d'Albert le Grand, parmi les œuvres sur les sympathies occultes et les amulettes dont il est question dans chapitre intitulé De ligaturis et suspensionibus lapidum. Celui-ci s'ouvre sur la première citation du chapitre 8 du De virtute universali d'Arnold Saxo, où il est question de la "vertu universelle occulte":

Ea vero quae ad hanc scientiam magis pertinere videntur, sunt ligaturae lapidum et suspensiones: quia in illis non nisi naturaliter ex virtutibus conferunt medicinam et iuvamen. De his igitur aliqua dicenda sunt ex Aristotele sumpta, et Constabulence, et Hermete Philosopho, et aliis quibusdam. Zeno autem in libro suo Naturalium quasi reddens rationem virtutis ligaturarum et suspensionum et ipsarum virtutum lapidum, dicit quod est virtus occulta universalis quae facit ex igne lapides, et similiter ex aqua quando funditur super locum qui vocatur bozon ${ }^{34}$.

445-452; Ullmann 1972, pp. 404-405. Sur la tradition arabe Ullmann 1975, pp. $196-200$.

31 On trouvera plusieurs exemples de ces traités dans Festugière 1950-1954, I, pp. 194-216, et dans Ullmann 1972, pp. 28-29 (et voir index à Sympathienbuch).

32 Ruska 1926, p. 188. Cf. Sirr al-halīqa 1979; Travaglia 2001.

33 Arnoldus Saxo, De floribus rerum naturalium, IV, 1 (cit. 8-11); IV, 3 (cit. 14 et 17-20); IV, 7 (cit. $3,15,16,28-30,33,36-40$ ); IV, 8 (cit. 1 et 2 ).

34 Albertus Magnus 1890, p. 55 [II, 3, 6]. Cf. Arnoldus Saxo, De floribus rerum naturalium, IV, 8 (cit. 1): "In libro de naturalibus Zeno: Virtus est occulta uni- 
Dans le mème ordre d'idées, dès le prologue à son De virtutibus lapidum (inclus dans le De floribus rerum naturalium), Arnold Saxo avait souligné que du mélange des éléments dans les pierres survenait une qualité irréductible aux qualités élémentaires:

Quia proprietas in lapidibus est que nulli complexioni est attributa. Sed, cum prima simplicia mixta sunt, et ex eis fit virtus una, sicut virtus attractiva in magnete, qui ferrum ex uno angulo trahit et ex alio angulo ipsum fugat, sic et virtutes specivoce sunt varie ac diversis gemmis lapidibus et eorum sigillis attribute ${ }^{35}$.

Cette théorie de la forme spécifique et de la vertu d'attraction qui en découle, dans le cas de l'aimant, introduisait à un catalogue minéralogique rassemblant dans un premier chapitre, les vertus des pierres. Ces dernières pouvaient opérer à distance, sous forme de talismans, en diffusant leur vertu lors du contact avec le corps par ligature; c'est l'objet du second livre (De sigillis) du De virtutibus lapidum, où les pierres sont présentées comme un lien entre le ciel et la terre par le biais de l'action thérapeutique ou magique des gravures de figures mythologiques ou astrologiques qu'elles portent. Le De virtutibus lapidum comptait parmi ses objectifs annoncés de rassembler les dires d'Aristote sur les pierres ${ }^{36}$. C'est aussi ce qu'a déclaré Albert le Grand, au seuil du De mineralibus qui s'intègre à son grand commentaire de la philosophie naturelle du Stagirite. N'ayant pas trouvé le "Livre des pierres d'Aristote", il avait dû se contenter d'extraits glanés ci ou là ${ }^{37}$. C'est la raison pour laquelle il

versalis que facit ex igne lapides, et ex aqua. Quando funditur ipsa super locum bezon, tunc coagulatur subito, nec amplius in suam materiam revertitur".

${ }^{35}$ Arnoldus Saxo, De floribus rerum naturalium, III, 1. Cf. Draelants 1993, p. 136.

36 Déjà, une longue notice sur l'aimant magnétique et sur les aimants végétaux et animaux se trouvait dans le 'livre des pierres' d'Aristote, justifiant l'exemple paradigmatique de l'aimant. Cf. Rose 1855 et Ruska 1912, qui présente les deux versions arabes subsistantes du 'lapidaire d'Aristote' et des extraits de la traduction hébraïque.

${ }^{37}$ Albert le Grand dit à plusieurs reprises n'être jamais parvenu à retrouver le lapidaire d'Aristote. Albertus Magnus 1890, p. 1 [I, 1, 1]: "De his autem libros Aristotelis non vidimus, nisi excerptos per partes. Et haec quae tradidit Avicenna de his in tertio capitulo primi sui libri quem fecit de his, non sufficiunt". Il fait allusion au De congelatione et conglutinatione (en réalité une partie du Shifä d'Avicenne, qui, une fois traduite, a été inclue à la fin des Météorologiques d'Aristote). A nouveau, il le déplore à p. 57 [II, 3, 6] et à p. 59 [III, 1, 1]. adopta le catalogue des pierres et le De sigillis d'Arnold Saxo comme source directe et principale du deuxième livre, aux deuxième et troisième traités ${ }^{38}$.

Mais quels sont les liens entre cette vertu d'attraction particulière et la "vertu universelle" ou la "vertu céleste"? Ils résident dans les relations analogiques microcosme-macrocosme qui fondent la loi naturelle et qui s'appliquent tant aux vertus données aux pierres qu'à toute créature terrestre, à travers une cascade de causes venues du ciel. Au début de ce deuxième livre, Albert se place au niveau général pour dire que la vertu occulte a été observée dans toute la nature par les "physiologues" (naturalistes), qu'elle est clairement reconnaissable en médecine et surtout dans la pratique des ligatures et des talismans minéraux, végétaux et animaux.

Sed tria sunt quae potissime sunt investiganda, virtutis eorum causa videlicet, et descriptio eorum sigillatim, et sigilla quae inveniuntur in quibusdam eorum depicta. His tribus habitis, non quaerimus amplius physice scire de lapidum natura. Causa autem virtutis lapidum occulta est valde, et varia in ea sensisse videntur multi physiologorum. Multi etiam dubitare videntur an insit lapidibus virtus aliqua de his quae videntur esse in eis, sicut curare anthraces, fugare venena ... Expertum est sapientissime: quoniam magnetem videmus ferrum attrahere, et adamantem illam virtutem in magnete restringere ... Nihil tamen est in universa natura quod non habeat propriam suae speciei operationem ... hoc autem in simplicibus probatur medicinis, et in scientia de incantatione et alligatione, ubi ostenditur membra diversorum animalium, aut collo, aut coxae, aut alii membro corporis hominis alligata, mirabiles operari effectus. Idem est de herbis, radicibus, et lignis ${ }^{39}$.

Ainsi, le soleil distribue sa "vertu universelle", dit Albert, dans la vertu de chaque pierre, comme c'est le cas pour l'escarboucle (carbunculus) qui brille plus que tout autre, ou pour le grenat (granatus).

Omnes autem virtutes infundi in inferioribus omnibus per circulum Alaur, quem primum circulum imaginum coelestium esse dicebant. Has autem virtutes descendere in res naturae nobiliter et ignobiliter ... Hanc igitur causam isti dicunt, quoniam lapides pretiosi prae

38 Arnold Saxo avait lui-mème utilisé le 'livre des pierres' d'Aristote en deux versions, l'une traduite par "Gérard" (probablement Gérard de Crémone), l'autre tirée de Dioscoride. Il y avait ajouté des citations tirées d'autres intermédiaires des dires d'Aristote. Cf. Draelants 2001, pp. 117-118.

${ }^{39}$ Albertus Magnus 1890, pp. 23-24 [II, 1, 1] 
aliis habent mirabiles virtutes: quia videlicet in substantia magis similantur superioribus, et in lumine et perspicuitate: propter quod a quibusdam eorum stellae elementales esse dicuntur lapides pretiosi ... Tertius est, qui vocatur ignescens et rutilans, qui est in Sole et Marte et in quibusdam aliis: et hunc primo quidem accipit carbunculus, et postea palachius sive palatius et granatus ${ }^{40}$ et quidam alii. Ideoque dicunt carbunculum nobilissimum et universaliter habentem virtutem omnium aliorum lapidum: quia Sol cuius virtutem similem accipit, nobilior est omnibus virtutibus caelestibus, et est sua virtus universalis dans lucem omnibus caelestibus et virtutem ${ }^{41}$.

Continuant à recenser les opinions philosophiques antiques dans sa recherche inlassable des causes rationnelles des phénomènes, Albert concilie plus loin en une phrase l'opinion d'Hermès avec les affirmations théoriques que nous avons déjà rencontrées chez Arnold Saxo, à propos de l'analogie, de la vertu spécifique et de la hiérarchie des causes descendant des cercles célestes vers la terre.

Omnibus tamen Antiquis probabilius dixit Hermes de causa virtutis lapidum: quia scimus pro constanti omnium inferiorum virtutes a superioribus descendere. Superiora enim substantia et lumine et situ et motu et figura influunt in inferioribus omnes nobiles virtutes quae sunt in ipsis. Tamen hoc dictum imperfectum est in physicis, licet forte in astronomicis et magicis esset sufficiens: quia in physicis dicitur causa quae est in materia operans: talis autem est elementalis aut qualitates elementorum prout sunt in commixtio, vel forma substantialis tales complexiones consequens ${ }^{42}$.

Mélangeant plusieurs sources d'information, dont Aristote, Avicenne, "Zénon" et Hermès, Albert le Grand et Arnold Saxo paraissent avoir identifié la virtus universalis à la vis celestis. Le principe hermétique de la vertu universelle venait ainsi compléter l'explication physique de type aristotélicien, qui ne suffisait plus. Les théories des quatre causes et de l'analogie, qui s'interpénètrent au XIII ${ }^{\mathrm{e}}$ siècle, ne satisfaisaient plus en physique et particulièrement en médecine. En métaphysique et en astrologie, la conception néoplatonisante de la hiérarchie des causes fut adoptée par les Latins via

40 Les mêmes liens entre ces pierres sont exprimés chez ${ }^{*}$ Arnold Saxo.

41 Albertus Magnus 1890, p. 24 [II, 1, 2]

42 Albertus Magnus 1890, p. 27 [II, 1, 3] le De causis pseudo-aristotélicien et le De proprietatibus elementorum également pseudépigraphe. Les contenus de ces traités se conjuguaient sans peine avec les notions hermétiques de l'identité d'essence (la vertu universelle du soleil) et de la différence d'être (la vertu spécifique d'une substance).

b) Quelques prolongements chez Thomas d'Aquin et dans l'ésotérisme de la Renaissance.

Les traités de magie de la Renaissance ont tiré parti de ces doctrines articulées autour de la forme spécifique et de la forme substantielle mais ils en ont fortement réduit le champ d'application. Des philosophes du $\mathrm{XV}^{\mathrm{e}}$ siècle comme Pic de la Mirandole ou Marsile Ficin ${ }^{43}$ se sont illustrés dans cette veine à propos des talismans. Une immense littérature, prenant la suite du Speculum astronomiae attribué à Albert le Grand, a disserté sur le caractère licite ou non de l'emploi des images astrologiques sur les talismans ou sous forme de statues ${ }^{44}$. Cornelius Agrippa de Nettesheim, formé à Cologne au tournant des $\mathrm{XV}^{\mathrm{e}}$ et $\mathrm{XVI} \mathrm{I}^{\mathrm{e}}$ siècles, s'inscrit dans la ligne de l'albertinisme. Il consacre plusieurs chapitres de son De occulta philosophia à la question des vertus des pierres ${ }^{45}$, inscrivant clairement les qualités occultes dans un cadre qui mêle idées platoniciennes, théorie aristotélicienne des causes et hermétisme ${ }^{46}$. On constate sans s'étonner que le De mineralibus authentique (en particulier le livre II) et le De mirabilibus mundi pseudo-albertinien en furent deux sources essentielles ${ }^{47}$.

Quoi qu'il en soit, le nom d'Hermès comme source d'inspiration à propos de la vertu propagée par la lumière du soleil semble avoir rapidement disparu. Son nom apparait cependant encore en 1598

43 Pour Ficin, voir Blum 1992, p. 56.

44 La question de la causalité astrale ayant été récemment examinée dans tous ses développements par Weill-Parot 1999 et Weill-Parot 2002, il est inutile d'y revenir.

${ }^{45}$ Cf. Hutchison 1982, pp. 239-240, qui semble dire que la définition de la propriété occulte (comme échappant aux quatre éléments de la cosmologie sublunaire d'Aristote et opposée aux qualités manifestes qui reflétaient les caractéristiques universelles des quatre éléments présentes dans tous les corps terrestres) n'est pas antérieure à la fin du $X V^{\mathrm{e}} \mathrm{s}$.

46 Agrippa 1992, pp. 107-110 [I, 11-13, cf. apparat des sources] reprend Albertus Magnus 1890, II, 1, 1, cité ci-dessus.

47 Perrone Compagni in Agrippa 1992, pp. 20-25 
dans le traité espagnol du pharmacien Gaspar de Morales, condamné par l'Inquisition. Dans le passage ci-dessous, largement inspiré d'Albert le Grand, sont réunis la plupart des éléments de l'explication que j'ai évoqués, ainsi que la notion d'expérience $^{48}$.

Aristoteles en el 1. lib. de los Fisicos donde dize, que de sola la substancia, o forma de la cosa, no se puede conocer la virtud, n atribuirsele ninguna accion, sino todo el mixto, porque aunque es verdad, que la forma es causa de las acciones no se entiende, que sea ella sola, sino todo el compuesto, aunque Alberto magno siguiendo el modo de filosofar natural, pone, que las virtudes de las piedras se hazen, y son causadas de la especie, o forma substancial de la piedra, porque como en el mixto ay algunas virtudes, que provengan de los elementos tan solamente como son la dureza ... las virtudes particulares, que en ellas ponemos, como (verbi gratia) que el Magnete ... Esto no proviene de las primeras qualidades, sino de la virtud celeste ... compuesto, influyendo el Cielo ... resulta al tiempo de su mixtion la virtud celeste ... Y assi porque el compuesto, la parte mas principal es la forma, se le suele atribuir toda la virtud del compuesto ... como dize Hermes Trimegisto. Adhuc En una misma especie de piedras suele aver variacion, porque la forma produzida en este mixto obra segun la disposicion de la materia, y del lugar, por la direccion, o obliquidad de los rayos del Sol, que corresponde ... vemos una piedra semejante a otra en todos los acidentes individuales, y no produzir el mesmo efeto que la otra, de donde considerando Alberto magno esto, atribuye a la experiencia, mediante su forma substancial de la piedra, que es todo del compuesto, y la forma, principalmente, a quien se le atribuyen pro excelencia las acciones.

Les théories explicitées à propos des pierres chez Albert le Grand connaîtront aussi des prolongements en dehors de la minéralogie, et particulièrement en métaphysique. La recherche des causes par l'observation de leurs effets était une des motivations principales des péripatéticiens, qui organisaient le monde à partir des causes universelles et des formes universelles abstraites de la diversité des accidents de la matière. Chez le théologien Thomas d'Aquin, l'idée de diffusion de la vertu solaire, prisée par les hermétistes, s'encadre dans un hylémorphisme platonicien et se fond dans la hiérarchie des causes issues du mouvement céleste. Les opérations occultes qui affectent les phénomènes de la nature sont clairement rattachées à

48 Gaspar de Morales 1977, pp. 191-192 [15]. une influence presque mécanique des corps célestes ${ }^{49}$. On ne parle cependant plus de "vertu universelle", il n'est plus question d'expérience, ni d'hermétisme: Thomas rapporte les opérations et modifications des corps mixtes qui ne peuvent être expliquées par les interactions des éléments à des principes supérieurs appelés altiora principia ou superiora agentia, qui sont les corps célestes ou, plus haut que ces derniers, des substances séparées à l'image des idées platoniciennes. La répercussion de ces causes hiérarchisées renvoie à la première, parfois lointaine: Dieu. Dans la Somme théologique, il se réfère au De generatione d'Aristote pour aborder la question de la vertu propre aux corps mixtes en fonction de leur forme substantielle ${ }^{50}$.

Comme ses prédécesseurs, il donne la plupart du temps comme exemple de propriété particulière (virtus) dans un corps mixte, celui de l'aimant. Ainsi, dans la quaestio disputata écrite en 1268, De spiritualibus creaturis, ou dans les Quaestiones quodlibetales écrites en $1272^{51}$. Cette manière d'envisager la causalité céleste pour des propriétés extraordinaires caractéristiques de certaines formes spécifiques est encore plus explicite dans la lettre De occultis operibus

49 Cf. Litt 1963, notamment pp. 121-122, pour la conception de la causalite céleste chez Thomas d'Aquin et la question de la vertu des mixtes.

${ }^{50}$ Blum 1992, p. 50, qui attribue la formation du concept de 'forme substantielle' à Thomas d'Aquin, cite Summa theologiae, I, 76, 4: "Et ideo dicendum est, secundum Philosophum in I De generat. $\{10,327 \mathrm{~b} 22\}$ quod formae elementorum manent in mixto non actu, sed virtute. Manent enim qualitates propriae elementorum, licet remissae, in quibus est virtus formarum elementarium. Et huiusmodi qualitas mixtionis est propria dispositio ad formam substantialem corporis mixti puta formam lapidis, vel animae cuiuscumque".

51 Litt 1963, pp. 121-122, cite De spirilualibus creaturis, II: "Forma enim elementi non habet aliquam operationem nisi quae fit per qualitates activas et passivas, quae sunt dispositiones materiae corporalis. Forma autem corporis mineralis habet aliquam operationem excedentem qualitates activas et passivas, quae consequitur speciem ex influentia corporis caelestis, ut quod magnes attrahit ferrum et quod saphyrus curat apostema"; Quaestiones quodlibelales, XII, 9, 2: "Dicendum quod aliquid potest habere virtutem aliquam et secundum propriam naturam et secundum virtutem superioris causae: verbi gratia, commixtum ex elementis habet virtutem unam ex natura elementorum secundum motum praedominantis elementi; aliam habet ex corpore caelesti, sicut quod adamas attrahit ferrum, quod non reducitur ad virtutem elementorum". D'autres passages sont mentionnés à propos de l'activité des mixtes. 
naturae, où Thomas considère que la forme substantielle est la source de l'activité naturelle ${ }^{52}$.

\section{Alchimie: Aristote, Avicenne et la vis mineralis.}

L'expression "vertu universelle" apparait encore dans un passage relatif aux pierres copié dans un manuscrit de médecine astrologique du début du XIV ${ }^{\mathrm{e}}$ siècle. Il s'agit du manuscrit Barcelona, Biblioteca de Catalunya, 634, qui contient des traductions de l'hébreu au latin ${ }^{53}$. On y lit ce qui se présente comme des extraits de l'ouvrage d'Aristote sur les pierres. Or, ceux-ci ne se retrouvent pas dans les témoignages connus sur cette œuvre, que ce soient les textes arabes du lapidaire d'Aristote ou les passages qui en sont conservés dans les œuvres arabes ou latines. Ils ne sont pas tirés non plus du De congelatione et conglutinatione lapidum d'Avicenne, qui constitue, dans la littérature scientifique du bas Moyen Âge, la fin du quatrième livre des Météorologiques d'Aristote; rien d'approchant non plus dans le Secretum secretorum pseudo-aristotélicien, en dépit de la référence qui précède le passage en question.

Il s'agit d'une définition de la virtus universalis conforme à celles que nous avons déjà rencontrées et qui s'applique directement à toute substance animale, végétale ou minérale, à travers le pouvoir des planètes.

Dicit Aristoteles in libro de secretis capitulo de proprietate lapidum quod gradus et dispositio planetarum et lapidum mineralium secundum essentiam recipit for $<$ mam $>$ vincentem et dominantem in eo secundum originem et naturam a superioribus naturis, unde vincens in plantis est natura aque et in mineralibus <et lapi>dibus vincens

52 Thomas Aquinas 1939, p. 192, $\mathrm{n}^{\circ}$ 6: "Quaedam vero operationes occultae in quibusdam inveniuntur corporibus, quae similiter inveniuntur in omnibus quae sunt eiusdem speciei, sicut omnis magnes attrahit ferrum"; p. 192, n 7 : "Talis autem virtus quae est talium actionum vel passionum principium, maxime ostenditur ex forma rei specifica derivari"; p. 194, n 11 : "Procedunt tamen tales formae a substantiis separatis ut primis principiis, quae mediante virtute et motu corporum caelestium imprimunt formas apud se intellectas in materiam corporalem"; p. 195, $\mathrm{n}^{\circ}$ 14: "Formae vero mixtorum, scilicet inanimatorum corporum, puta lapidum", "praeter virtutes et actiones quas ab elementis participant ex quibus componuntur: quasdam alias virtutes et actiones nobiliores habent consequentes formas eorum specificas".

53 Ce texte m'a été signalé par Charles Burnett, que je remercie vivement pour cette information. est natura terrea. Recipit ergo natura planetarum extensionem de natura aquarum sicut aqua recipit extensionem per <impe>tum et impulsionem ventorum in suo loco, et sicut aqua est diversarum figurarum, quia in aqua sunt multe figure, sic accidit in plantis <quod> omnis figure inveniuntur in plantis. Cum itaque vincens in plantis sit aqua et non extenditur nisi per difusionem, et operator $\mathrm{d}<$ iso>lucionis vel difusionis aquarum sit perpetuus et incessanter operans in suo celo, scilicet Mercurius, quomodo universaliter verum est quod unusquisque <pla>neta regit et disponit quod convenit et asimilatur sue nature. Verbi gratia, Saturnus tenet terram, Mercurius aquam, Iupiter aerem, <Mars> ignem, et non reperitur hec conveniencia in operibus planetarum vel ex eorum corporibus sed in oppositionibus vel operacionibus quod semper habent continuas virtutes perpetuas per superiorem virtutem universalem que est super omnes virtutes istarum operacionum etc.

Vu le contexte du manuscrit, il ne serait pas étonnant que ce passage soit traduit directement de l'hébreu. Les citations du Livre des pierres d'Aristote, mais aussi les extraits de Zénon, Belbetus (Balīnūs) et Pythagore qui constituent une part du De virtute universali chez Arnold Saxo, contiennent également des termes issus de cette langue ${ }^{54}$. Malheureusement, la transmission de bribes du Livre des pierres d'Aristote en hébreu est encore quasiment inconnue.

Comme le souligne ce texte inédit, parmi les règnes naturels, celui des corps mixtes et des pierres en particulier est un terrain privilégié pour la théorie de la "diffusion" d'une "vertu universelle". Elle s'applique à l'explication des vertus propres aux pierres, mais aussi à la transformation des métaux et matériaux intermédiaires, c'est-àdire à l'alchimie. Dans cette discipline, Albert le Grand, à nouveau, fait appel à elle pour adapter la théorie des causes dans le but d'élucider la transformation chimique des métaux.

Quod enim virtutes elementales et caelestes faciunt in vasis naturalibus, hoc faciunt in vasis artificialibus ... et quod facit natura calido Solis et stellarum, hoc faciet et ars calido ignis ... huic enim caelestis inest virtus quae primo commiscuit eam: et haec inclinatur ad hoc vel ad illud per artis iuvamen. Caelestis enim virtus valde communis est, et accipit determinationem per virtutes eorum quae sunt subiectum eius in rebus commixtis: hoc enim modo virtutes caelestes operari videmus in tota natura generatorum, maxime in his quae ex putrefactione generantur. In his enim videmus

${ }^{54}$ Cf. Draelants 2000b, pp. 198-199. 
virtutes stellarum influere virtutes in id ad quod convenientiam habet materia ${ }^{55}$.

Albert explique que les vertus élémentaires se mélangent aux vertus célestes dans les vases des alchimistes; les vertus célestes, émanant des étoiles, sont ainsi mêlées à toute la nature des choses générées et $\mathrm{y}$ opèrent. L'alchimie est donc capable de changer la substance des métaux et les propriétés typiques de leur espèce, en utilisant artificiellement la vertu céleste présente en toute chose par l'intermédiaire de l'élément chaleur.

Dans un autre passage du De mineralibus relatif à la génération des pierres, on se trouve confronté d'autre part à la vis mineralis, analogue à la vis formativa. Albert le Grand a emprunté ici à Arnold Saxo des extraits du lapidaire attribué à Aristote, mais aussi des Météorologiques authentiques. Or, le quatrième livre de la version latine des Météorologiques contient trois chapitres de type alchimique ajoutés par Alfred de Shareshill. Ils concernent en particulier la formation des montagnes et des pierres et sont tirés du Shifa d'Avicenne; ils ont circulé sous le nom de De congelatione et conglutinatione lapidum. Il y est bien question de la "force qui lapidifie", la vis mineralis, "quwwa ma'diniyya" en arabe ${ }^{56}$ :

Virtus enim mineralis quaedam communis virtus est efficiens et lapides et metalla, et ea quae sunt media inter haec ... Et quia propria nomina huius virtutis non habemus, ideo per similia oportet declarare quae sit illa virtus. Dicamus igitur quod sicut in semine animalis quod est superfluum nutrimenti, descendit a vasis seminariis vis formativa animalis, quae format et efficit animal, et est in semine per modum illum quo artifex est in artificiato quod facit per artem: sic est etiam in materia aptata lapidibus virtus formans et efficiens lapides et producens ad formam lapidis huius vel illius ${ }^{57}$.

Faut-il confondre cette vis mineralis avec la vis celestis ou virtus universalis? Théoriquement non, en dépit d'un extrait du De mineralibus cité plus haut, où certains manuscrits - ainsi que l'édition de A. Borgnet - ont substitué minerali à universali à propos de

55 Albertus Magnus 1890, p. 71 [III, 1, 9].

56 Cf. Avicenna 1965, p. 3. Merci à Cécile Bonmariage pour son aide à la compréhension des passages arabes d'A vicenne.

57 Albertus Magnus 1890, p. 7 [I, 1,5]. l'ouvrage d'Hermès sur la vertu universelle ${ }^{58}$. Ce qui apparaît à première vue comme une leçon due à une faute de copiste signifie davantage: le fait révèle qu'il y a eu intersection entre deux sources, avec pour conséquence de fusionner deux idées proches sur la génération des pierres. Dans le De mineralibus I, 1, 4 la vis mineralis contribue à expliquer la formation des pierres et des montagnes, alors que la virtus universalis intervient en II, 1, 2 pour diffuser la vertu supplémentaire, thérapeutique ou magique, exercée par les pierres. L'intersection entre ces concepts a lieu dans un contexte de magie naturelle, de sympathie universelle, de liens microcosme/ macrocosme; elle est réalisée grâce à la figure du philosophe et magicien Hermès. Les deux notions ont, à l'insu de leurs utilisateurs, un mème véhicule textuel: Avicenne, une fois alchimiste (vis mineralis), une fois médecin (vis universalis), mais elles sont rapportées à des questions de philosophie naturelle liées au corpus des textes aristotéliciens.

Le concept qui relie la vertu universelle de la chaleur et du soleil en particulier d'une part, et la force lapidificative d'autre part, est la vis formativa, la force formative. Elle se trouve déjà dans le $D e$ natura loci d'Albert le Grand, où il l'associe avec les dires d'Hermès "sur les vertus universelles" et souligne qu'elle ne tient pas à la matière:

Convenientia tamen est in locis, quae vicina sunt in proprietatibus communibus, et ideo ea quae vicine habitant, similes frequenter habent complexiones et similia conservantia et similia corrumpentia in communi. Similitudo autem et dissimilitudo locorum et virtutum formativarum, quae sunt ex loco, in materia per signum maxime deprehendi potest in quodam magnetis genere, qui in uno angulo fugat ferrum et in alio attrahit ipsum; cum enim unus angulus ab alio non distet per magnum loci spatium, oportuit, quod vicina valde loca aliquando sint diversarum et contrariarum virtutum; haec enim contrarietas non est ex materia, quia materia non est causa virtutis et formae. Oportet igitur, quod sit ex loco informato a figuratione radiorum stellarum. Et hoc est, quod egregie dicit Hermes in libro de virtutibus universalibus, quod constellatio est causans virtutem qualitativam stellarum, quae infunditur inferioribus et est formativa ipsorum per qualitates elementorum, quae sunt sicut instrumenta virtutum caelestium ${ }^{59}$.

58 Voir ci-dessus, p. 167: "Hermes autem in libro quem de minerali (lege universali) virtute scribit"

${ }_{59}$ Albertus Magnus 1987, pp. 8, $78-9,12[1,5]$. 
Cette vis formativa, observée chez les animaux et les végétaux, était déjà alléguée dans son premier ouvrage de philosophie naturelle, la Summa de creaturis; à propos des pierres, il disait qu'elle provenait de la vertu céleste et du mouvement céleste et la reliait à la forme substantielle. Du mélange des éléments dans chaque minéral résultait leur forme substantielle, d'où provenait une force formative aidée de la force céleste:

Sicut illa, quae generantur ex putrefactione vel commixtione elementorum sola, sicut est generatio mineralium, est virtus formativa respectu formae substantialis a virtute caelesti tantum. Generatio autem elementorum est a motu coeli cum virtute caelesti, quae est respectu formae substantialis elementi. Et est ratio Aristotelis haec, quod formae illa substantiales omnes aut generantur ex se aut non ${ }^{60}$.

La confusion est ici réalisée entre les deux registres vis formativa générale / vis mineralis particulière d'une part, et virtus universalis générale / virtus specifica particulière d'autre part, et semble avoir existé dès les premiers temps de la diffusion des Météorologiques chez les Latins. Dans la première partie du De floribus rerum naturalium d'Arnold Saxo, relative à la cosmologie ${ }^{61}$, lui ou sa source, probablement influencés par l'existence d'un De virtute universali hermétique, ont substitué vis universalis à vis mineralis dans les citations d'Avicenne qui ont circulé avec les Météorologiques, comme le montrent les extraits ci-dessous ${ }^{62}$.

\begin{tabular}{|c|c|}
\hline $\begin{array}{l}\text { De floribus rerum naturalium I, } \\
\text { De celo et mundo, } \mathrm{V}, 2 \text { [cit. } 6,8]\end{array}$ & $\begin{array}{c}\text { De congelatione et conglutinatione } \\
\text { lapidum }{ }^{63}\end{array}$ \\
\hline $\begin{array}{l}\text { Fit etiam generatio montium sicut } \\
\text { generatio lapidum, quia aqueductus } \\
\text { addit illis lutum unctuosum continue. } \\
\text { Per longitudinem temporis desicca- } \\
\text { tur, et fit lapis. Et postea vis univer- } \\
\text { salis vertens aquas in lapides. Et ideo } \\
\text { in multis lapidibus inveniuntur que- } \\
\text { dam partes animalium aquaticorum } \\
\text { et aliorum. }\end{array}$ & $\begin{array}{l}\text { pp. 48-49: fit eciam generacio mon- } \\
\text { cium sicut lapidum quia aqueductus } \\
\text { adducit lutum unctuosum continue } \\
\text { quod longitudine temporis [per longi- } \\
\text { tudinem temporum] dessicatur et fit } \\
\text { lapis sed vis mineralis brevius [om.] } \\
\text { vertit [vertens] aquam in lapides. Et } \\
\text { ideo in multis lapidibus inveniuntur } \\
\text { partes quedam quorundam animalium } \\
\text { aquaticorum et aereorum [aliorum]. }\end{array}$ \\
\hline
\end{tabular}

60 Albertus Magnus 1895, p. 315 [1, 1, 1, 6 ].

${ }^{61}$ Le texte est édité d'après les deux manuscrits subsistant pour cette partie: Erfurt, Wissenschaftliche Allgemeinbibliothek, Ampl. $8^{\circ}$ 77, et Oxford, Bodleian Library, Lat. misc. e. 34

62 Il reste à faire une enquête sur les manuscrits contenant l'appendice au IV ${ }^{\mathrm{e}}$ livre des Météorologiques, pour apprécier le poids respectif des leçons mineralis contre universalis dans la tradition manuscrite.

${ }^{63}$ Entre crochets, les autres leçons de l'apparat d'Holmyard-Mandeville dans Avicenna 1927
Sepe etiam fiunt lapides ex igne, cum extingitur. In ripis Geon visa est terra, que commemoratur in lapidem converti in spatio viginti trium annorum. Panis quoque corathym in lapidem conversus est, remansit enim ei color suus. Similiter predicta vegetabilia, et quedam animalia vertuntur bilia, et quedam anima vertuntur in lapides virtute qua lapidificata, et fit in loco lapidoso, et discontinuantur subito virtute quadam, que exit a terra in hora terre motus, que convertit in lapides.

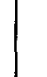

p. 47: Sepe eciam fiunt lapides ex igne cum extinguitur - p.45: In ipso [ripis] quoque Gion visa est terra que dicitur in lapidem commuti [converti] in spacio annorum centum [23 dans la version arabe] - p. 47: Panis quoque prope toracem in lapidem conversus est remans tam lapide conversus est re torace color suus, - p. 46. similiter quoque quedam vegetabilia et quedam animalia vertuntur in lapides virtute quadam minerali lapidificativa et fit in loco lapidoso vel discontinuantur subito virtute quadam que exit a terra in hora terre motus que convertit lapides quod consequitur in hora illa.

Suntque aque seorsum accepte non congelantur. Que si prope alveum suum fundantur, congelantur fiuntque lapides. Scimus, quod in terra illa est vis universalis, que congelat aquas in principia lapidum. pp. 45-46: Suntque aque que seorsum accepte non conglutinantur que si prope alveum suum fundantur congelantur fiuntque lapides. Scimus quoque quod in terra illa est uisi uistal [vis mineralis] que congelat ipsam (aquas). Principalia quoque ipsam (aquas). Principalia quoque

A la même époque à peu près, la Summa philosophiae attribuée à Robert Grosseteste tente d'expliquer le pouvoir des pierres précieuses. L'auteur y développe l'idée de la "forme spécifique" et de la "forme substantielle". Il répète que la force intrinsèque trouvée dans une pierre est causée par l'espèce même de la pierre et par la forme substantielle. Pour ce faire, l'auteur reprend à son compte les développements du De mineralibus d'Albert le Grand déjà évoqués à propos des vertus des pierres et de leurs sceaux pour un usage talismanique; du même coup, il en adopte toutes les références aux sources et aux auctoritates, parmi lesquelles, Hermès ${ }^{64}$ :

Virtutes mirabiles inesse lapidibus maxime pretiosis certissimum est. Utrum tamen a primis corporibus id est elementis, seu qualitatibus activis et passivis insint eis, sicut asserit Alexander, an a motoribus orbium, sicut videtur Avicennae, an a virtutibus caelorum corporalibus, sicut Hermes ac Ptolomeus sensisse videtur, an ab anima aliqua, sicut Democritus totaque Pythagoraeorum schola opinatur, an ab idea, sicut Plato aestimavit, an forte ab ipsa lapidis specie formaque substantialis, sicut Constantinus aliique

64 Robertus Lincolniensis 1912, p. 633 [XIX, 6]. 
plures affirmant, sine magna contradictione difficile est definire. Et videtur Alberto Coloniensi, qui certius in hac materia locutus est ceteris, quod a forma consequente speciem causetur ad virtutes caelestes tam motorum, quam orbium et quadraginta octo imaginum in astris vel signorum ex una parte, ad naturam complexionatam a miscibilibus causatam ex altera, atque ita, cum sit species lapidis cuiusque unicum quid in se ... Itemque ex varietate nobilitatis et ignobilitatis materiae erit differentia virtutis in individuis specierum secundum fortius et debilius, numerosius et minus numerose, quod testatur Hermes.

4. Pour conclure.

La fortune 'littérale' de l'expression latine virtus universalis semble avoir émergé, mais aussi s'être limitée à quelques textes de philosophie naturelle du XIII ${ }^{\mathrm{e}}$ siècle, essentiellement chez Arnold Saxo et chez Albert le Grand. En revanche, le concept lui-même a fleuri et prospéré sur un terrain où des notions voisines avaient déjà leur place et leurs champs sémantiques se sont en partie confondus dans le contexte philosophique 'pluridisciplinaire' de l'époque. Dans l'appendice aux Météorologiques, on a ainsi observé un certain recouvrement, aggravé par la tradition manuscrite, entre vis mineralis et virtus universalis; il demeura chez les naturalistes, qui cependant empruntaient en conscience l'une et l'autre notion à des sources distinctes. Mais surtout, la "vertu universelle", à la fois responsable des qualités spécifiques en médecine et en minéralogie, et véhicule de la causalité céleste en cosmologie, astrologie et même métaphysique, s'est trouvée sur le terrain de notions existantes comme la "substance toute entière", la "forme spécifique" et la "propriété occulte", sans parler de la "force céleste".

L'utilisation du concept est typique du mélange doctrinal aristotélicien, néo-platonicien et hermétique, propre au caractère particulier des sources de la philosophie naturelle à l'époque. La rationalité scolastique a vu l'intérêt d'une doctrine qui permettait d'expliquer des phénomènes physiques jusque là impossibles à justifier par la théorie des éléments. De pair avec des concepts philosophiques apparentés, la notion de virtus universalis, relative aux 'qualités occultes', s'est répandue dans la littérature philosophique et scientifique du XIII ${ }^{\mathrm{e}}$ au XV $\mathrm{XV}^{\mathrm{e}}$ siècles, à la faveur de l'attrait pour la magie naturelle, la médecine et la science expérimentale, mais aussi en prolongement de certaines théories métaphysiques de la causalité Le corpus de philosophie naturelle arabo-latin s'est constitué à partir des cuvres d'Aristote traduites au XII ${ }^{\mathrm{e}}$ siècle, mais aussi des commentaires et traités arabes qui les accompagnaient. La notion étudiée ici en provient; elle s'avère avoir une triple origine, l'une aristotélicienne (ou pseudo-aristotélicienne, ce qui revient au même pour les auteurs de l'époque), l'autre avicennienne, et la troisième, hermétique.

Un des axes réside dans la diffusion rapide et large du Canon médical d'Avicenne. En quelque sorte, Avicenne a élargi la théorie causale aristotélicienne qui n'envisageait que des causes universelles en y ajoutant la possibilité de la cause cachée. Arnold Saxo l'assimila dans son livre appelé De virtute universali, où il reconnait aux naturalia une forme spécifique qui fait leur identité, qu'il s'agisse d'êtres animés ou inanimés; au-delà de leur complexion et du mélange des éléments simples, une "vertu" se manifeste à travers leurs propriétés caractéristiques: médicales, magiques, alchimiques Arnold reprend la même théorie en exergue de son lapidaire, consacré aux propriétés magnétiques et thérapeutiques des minéraux.

L'hermétisme a aussi favorisé l'assimilation de la doctrine, puisqu'il permettait d'ajouter la notion de Révélation pour les choses imperceptibles par les sens ou incompréhensibles par l'intellect. Grâce aux explications ajoutées par Albert le Grand dans le De mineralibus à partir d'une information similaire à celle d'Arnold, on comprend que c'est la vertu universelle qui, se diffusant dans chacun des êtres naturels, leur communique en quelque sorte sa force. La "vertu universelle" apparait avoir été le sujet d'un ouvrage hermétique non signalé jusqu'ici, mais clairement intitulé De virtutibus universalibus par Albert dans plusieurs de ses œuvres. On ne peut, dans l'état actuel des recherches, l'assimiler au De sex rerum principiis qu'Albert connaît et cite par ailleurs, mais la lumière du soleil, appelée natura universalis, potestas superiorum ou encore vigor universalis, y joue également ce ròle de force diffusée à travers les sphères célestes jusqu'aux plus petits êtres. Ce principe hermétique, allié à la métaphysique aux accents théologiques que le De causis pseudo-aristotélicien a livrée à l'Occident, a débouché sur une conception hiérarchisée de la causalité céleste qui fera les belles pages des ouvrages sur les sceaux des pierres et les images astrologiques dotées de pouvoir céleste. Il s'est aussi mêlé à l'explication aristoté- 
licienne des quatre causes - première, matérielle, efficiente, et formelle où intervient la vertu spécifique - qui président à la formation des pierres, entre autres dans les Météorologiques.

Enfin, pour expliquer la transformation des minéraux et métaux par la nature, mais aussi par l'artisan, Albert le Grand a puisé son argumentation philosophique dans le De congelatione et conglutinatione lapidum d'Avicenne. Ainsi, le principe de formation des pierres ou la cause formelle, c'est-à-dire la vis formativa ou la vis mineralis, rejoint la virtus universalis qui descend du ciel pour transmettre l'influence des astres. Tout apparaît ainsi mû par la vertu céleste en premier lieu ${ }^{65}$.

"Dicta vero Trismegisti non difficile est ad dicta Aristotelis reducere", dit Albert le Grand dans son commentaire au De causis ${ }^{66}$. Là se trouvait au XIII ${ }^{\mathrm{e}}$ siècle le noud de la question: avides de commenter 'tout Aristote', les philosophes de la nature ont dù bien souvent suppléer aux textes authentiques du philosophe par excellence. Ils furent contraints de compléter sa science en fonction de la transmission du corpus vetustius d'Aristote chez les Latins: soit en acceptant pour authentiques des textes pseudépigraphiques qui correspondaient bien à ce qu'Aristote aurait pu ou avait dû écrire, comme le Livre des pierres ou le De causis ou le De proprietatibus elementorum ou les chapitres alchimiques avicenniens des Météorologiques, soit en recourant à d'autres "philosophes antiques", comme Platon, Pythagore, Balīnūs ou Hermès, le "plus ancien philosophe païn”.

65 Il faut souligner que l'application de ces doctrines aux talismans ou images astrologiques et à l'alchimie des métaux aura pour autre conséquence la distinction nécessaire entre l'opération per artem et la génération per naturam, entre les choses fabriquées de la main de l'homme et celles issues sans transformation de la nature. La sculpture des pierres est donc considérée comme ayant un fondement naturel; l'action des pierres est le produit d'un processus causal qui obéit aux lois du destin, tandis que la magie naturelle est acceptée comme une science de la connaissance du réel. Cette distinction nature/artifice obligera à recourir de manière subtile à une succession des causes où l'homme n'est qu'un 'passeur de relais' dans la causalité astrale (Weill-Parot 1999; Weill-Parot 2002, pp. 301-316, pp. 877-878 et passim). Elle modifiera également l'attitude face à la magie 'naturelle' et à son revers, la magie nécromantique (cf. Lucentini 2000).

${ }^{66}$ Albertus Magnus 1993, p. 51, 58/59 [I, 4, 6]
Bibliographia

Agrippa 1992 = Cornelius Agrippa de Nettesheim, De occulta philosophia libri tres, ed. V. Perrone Compagni (Studies in the History of Christian Thought 48), Brill, Leiden-New York-Köln 1992.

Albertus Magnus 1867 = Albertus Magnus, De vegetabilibus libri VII, ed. E. Meyer - C. Jessen, Reimer, Berlin 1867.

Albertus Magnus $1890=$ Albertus Magnus, De mineralibus, ed. A. Borgnet (Opera omnia V), Vives, Paris 1890

Albertus Magnus 1895 = Albertus Magnus, Summa de creaturis, ed. A. Borgnet (Opera omnia XXXIV), Vives, Paris 1895.

Albertus Magnus 1973 = Albertus Magnus (pseudo-), The Book of Secrets of the Virtues of Herbs, Stones and Certain Beasts; also, A Book of the Marvels of the World, ed. M. Best - F. H. Brightman, Clarendon Press, Oxford 1973

Albertus Magnus 1980 = Albertus Magnus, De natura loci, ed. P. Hossfeld (Opera omnia, ed. Coloniensis V.2), Aschendorff, Münster 1980.

Albertus Magnus 1987 = Albertus Magnus, Physica. Libri I-IV, ed. P. Hossfeld (Opera omnia, ed. Coloniensis IV.1), Aschendorff, Münster 1987.

Albertus Magnus 1993 = Albertus Magnus, De causis et processu universitatis a prima causa, ed. W. Fauser (Opera omnia, ed. Coloniensis XVII.2), Aschendorff, Münster 1993.

Arnaldus de Villanova 1495 = Arnaldus de Villanova, Speculum medicinae, Martin Landsberg [Lipsiae 1495 ca.]

Arnaldus de Villanova 1509 = Arnaldus de Villanova, Speculum medicinae, in Opera omnia, Franciscus Fradin, Lugduni 1509, ff. 1-40.

Arnoldus Saxo 1905 = Arnoldus Saxo, Liber de floribus rerum naturalium, ed. E. Stange, Die Encyklopädie des Arnoldus Saxo, zum ersten Mal nach einem Erfurter Codex, Königliches Gymnasium zu Erfurt, Erfurt 1905.

Avicenna 1507 = Avicenna, Liber Canonis, Venetiis 1507

Avicenna 1927 = Avicenna, Kitāb al-Shifā $/$ De congelatione et conglutinatione lapidum, ed. \& tr. E. J. Holmyard - D. C. Mandeville, Avicennae 'De congelatione et conglutinatione lapidum' Being Sections of the Kitäb alShifa. The Latin and Arabic Texts Edited with an English Translation of the Latter and with Critical Notes, Geuthner, Paris 1927.

Avicenna 1965 = Ibn Sīnā, Al-Shifā. La physique. V. Les Métaux et la Météorologie (Al-ma'ādin wa' l-ațār al-'ulwiyya), ed. 'A. Montasir, Le Caire 1965 . 
Blum $1992=$ P. R. Blum, Qualitates occultae: Zur philosophischen VorgeBlu schichte eines Schlüsselbegriffs zwischen Okkultismus und Wisten (ed.), Die okkulten Wissenschaften in der Renaissance (Wolfenbütteler Abhandlungen zur Renaissanceforschung 12), Harrassowitz, Wiesbaden 1992, pp. 45-64.

Dales $1972=$ R. C. Dales, Marius 'On the elements' and the Twelfth Century Science of Matter, "Viator", 3 (1972), pp. 191-218.

De sex rerum principiis $1955=$ Liber Hermetis Mercurii Triplicis de VI rerum principiis, ed. Th. Silverstein, "Archives d'Histoire Doctrinale et Littéraire du Moyen Àge", 22 (1955), pp. 217-302.

Draelants 1992 = I. Draelants, Une mise au point sur les cuvres d'Arnoldus Saxo (1 $1^{\text {ere }}$ partie), "Bulletin de Philosophie Médiévale", 34 (1992) pp. $163-180$

Draelants $1993=\mathrm{I}$. Draelants, Une mise au point sur les auvres d'Arnoldus Saxo (2 $2^{e m e}$ partie), "Bulletin de Philosophie Médiévale", 35 (1993), pp. $130-149$.

Draelants 2000a $=I$. Draelants, Un encyclopédiste méconnu du XIII siècle: Arnold de Saxe. Oeuvres, sources, réception (Diss.), Université Catholique de Louvain, Louvain-la-Neuve 2000

Draelants $2000 \mathrm{~b}=\mathrm{I}$. Draelants, Le dossier des livres sur les animaux et les plantes de Iorach: tradition occidentale et orientale, in I. Draelants - A. Tihon - B. Van den Abeele (edd.), Occident et Proche-Orient. Contacts scientifiques au temps des croisades. Actes du Colloque de Louvain-laNeuve, 24-25 mars 1997 (Réminisciences 4), Brepols, Louvain-la-Neuve - Turnhout 2000, pp. 191-276.

Draelants $2001=\mathrm{I}$. Draelants, Introduction à l'étude d'Arnoldus Saxo et aux sources $d u$ 'De floribus rerum naturalium', in C. Meier-Staubach (ed.), Die Enzyklopädie im Wandel vom Hochmittelalter zur frühen Neuzeit. Internationales Kolloquium des Teilprojekts $D$ des SFB 231 der Universität Münster, 04.-07.12.1996 (Münstersche Mittelalter-Schriften 78), Fink, Münster 2001, pp. 85-121.

Eamon $1985=\mathrm{W}$. Eamon, Books of Secrets in Medieval and Early Modern Science, "Sudhoffs Archiv", 69 (1985), pp. 24-49.

Eamon $1994=\mathrm{W}$. Eamon, Science and the Secrets of Nature. Books of Secrets in Medieval and Early Modern Culture, Princeton University Press, Princeton (N. J.) 1994.

Festugière 1950-1954 = A.-J. Festugière, La Révélation d'Hermès Trismégiste, Gabalda, Paris 1950-1954.

Ganszyniec 1920-1921 = R. Ganszyniec, Studien $z u$ den Kyraniden, "Byzantinisch-neugriechische Jahrbücher", 1 (1920), pp. 353-369; 2 (1921), pp. 56-65 et 445-452.
Gaspar de Morales 1977 = Gaspar de Morales, De las virtudes y propriedades maravillosas de las piedras preciosas, ed. J. C. Ruiz Sierra, Gaspar de Morales, De las virtudes y propriedades maravillosas de las piedras preciosas. Prologo, introduccion y comentarios, Editora Nacional, Madrid 1977.

Hutchison 1982 = K. Hutchison, What Happened to Occult Qualities in the Scientific Revolution?, "Isis", 73 (1982), pp. 233-253.

Jacquart 1998 = D. Jacquart, La médecine médiévale dans le cadre parisien, Fayard, Paris 1998.

Jeck $1994=\mathrm{U}$. R. Jeck, Materia, forma substantialis, transmutatio Frühe Bemerkungen Alberts des Grossen zur Naturphilosophie und Alchemie, "Documenti e studi sulla tradizione filosofica medievale", 5 (1994), pp. 207-240.

Kyraniden 1976 = Die Kyraniden, ed. D. Kaimakis (Beiträge zur Klassischen Philologie 76), Hain, Meisenheim am Glan 1976.

Kyranides 1942 = Cyranides, ed. L. Delatte, Textes latins et vieux français relatifs aux Cyranides (Bibliothèque de la Faculté de Philosophie et Lettres de l'Université de Liège 93), Faculté de Philosophie et LettresDroz, Liège-Paris 1942, pp. 3-206.

Litt $1963=\mathrm{T}$. Litt, Les corps célestes dans l'univers de saint Thomas d'Aquin (Philosophes médiévaux 7), Nauwelaerts, Louvain-Paris 1963.

Lucentini $2000=$ P. Lucentini, L'ermetismo magico nel secolo XIII, in M. Folkerts - R. Lorch (edd.), Sic itur ad astra. Studien zur Geschichte der Mathematik und Naturwissenschaften. Festschrift für den Arabisten Paul Kunitzsch zum 70. Geburtstag, Harrassowitz, Wiesbaden 2000, pp. 409-450.

Pingree $1987=$ D. Pingree, The Diffusion of Arabic Magical Texts in Western Europe, in B. Scarcia Amoretti (ed.), La diffusione delle scienze islamiche nel Medio Evo europeo, Accademia Nazionale dei Lincei, Roma 1987, pp. 57-102.

Robertus Lincolniensis 1912 = Robertus Lincolniensis (pseudo-), Summa philosophiae, in Die philosophischen Werke des Robert Grosseteste, Bischofs von Lincoln, ed. L. Baur (Beiträge zur Geschichte der Philosophie des Mittelalters 9), Aschendorff, Münster 1912, pp. 275-643.

Rose 1855 = V. Rose, Aristoteles De lapidibus und Arnoldus Saxo, "Zeitschrift für deutsches Altertum", 18 (1855), pp. 321-455.

Ruska 1912 = J. Ruska, Das Steinbuch des Aristoteles, Heidelberg 1912.

Ruska 1926 = J. Ruska, 'Tabula smaragdina'. Ein Beitrag zur Geschichte der hermetischen Literatur (Heidelberger Akten der Von-Portheim-Stiftung 16), Carl Winter's Universitätsbuchhandlung, Heidelberg 1926. 
Sannino $2000=$ A. Sannino, Ermete mago e alchimista nelle biblioteche $d$ Guglielmo d'Alvernia e Ruggero Bacone, "Studi Medievali", 41 (2000), pp. 151-189.

Siraisi $1981=$ N. G. Siraisi, Taddeo Alderotti and his Pupils. Two Generations of Italian Medical Learning, Princeton University Press, Princeton (N. J.) 1981

Sirr al-halīqa 1979 = Balīnūs al-hakīm, Sirr al-halìqa wa san'at al-tabít $a$. Kitäb al-'ilal / Pseudo-Apollonios von Tyana, Buch über das Geheimnis der Schöpfung und die Darstellung der Nalur. Buch der Ursachen, ed. U. Weisser, Institute for the History of Arabic Science, University of Aleppo, Aleppo 1979.

Thomas Aquinas 1939 = Thomas Aquinas, De occultis operibus naturae, ed. J. B. McAllister, The Letter of Saint Thomas Aquinas De Occultis Operibus Naturae ad Quemdam Militem Ultramontanum. A Dissertation, Washington 1939.

Thorndike 1923-1958 = L. Thorndike, A History of Magic and Experimental Science, Columbia University Press, New York 1923-1958.

Travaglia 2001 = P. Travaglia, Una cosmologia ermetica, il Kitāb sirr alhalìqa / 'De secretis naturae', Liguori, Napoli 2001.

Ullmann $1972=$ M. Ullmann, Die Natur- und Geheimwissenschaften im Islam (Handbuch der Orientalistik I, Der Nähe und der Mittlere Osten VI.2), Brill, Leiden 1972

Ullmann 1975 = M. Ullmann, Die arabische Überlieferung der Kyranis des Hermes Trismegistos, in F. Rundgren (ed.), Proceedings of the $\mathrm{VI}^{\text {th }}$ Congress of Arabic and Islamic Studies, Stockholm, 1972, Stockholm 1975, pp. 196-200.

Weill-Parot $1999=\mathrm{N}$. Weill-Parot, Causalité astrale et 'science des images' au Moyen Age: éléments de réflexion, "Revue d'histoire des sciences", 52 (1999), pp. 207-240.

Weill-Parot $2002=$ N. Weill-Parot, Les 'images astrologiques' au Moyen Âge et à la Renaissance. Spéculations intellectuelles et pratiques magiques, $X I I^{e}-X V^{e}$ siècle (Sciences, techniques et civilisations du Moyen Âge à l'aube des lumières 6), Champion, Paris 2002.

Wilcox 1995 = J. Wilcox, Qustā ibn Lūqā's 'Physical Ligatures' and the Recognition of the Placebo Effect, with an Edition and Translation, "Medieval Encounters", 1/1 (1995), pp. 1-50. 